The application of joints which can be disassembled is studied within the framework of the development of high current $(25 \mathrm{kA})$, high power $(0.5 \mathrm{~kW})$ cryogenic current supplies (flux pumps). ${ }^{1,2}$ The behaviour of a scale model with currents up to $5 \mathrm{kA}$ will be presented here.

Several means of diminishing the joint resistance have been researched with special attention being paid to detection methods to measure the resistance, and their accuracies.

The role of the solder has been investigated. A simple model gives good qualitative understending of the matter.

The presence of ac currents in a cryogenic current supply causes considerably higher losses in the joint because of the non-homogeneous distribution of the current. A good impression of this distribution cen be obtained by measuring the field along the joint. The measured results are in good agreement with calculated ones. $A$ useful length of the joint under ac conditions can be defined and is helpful for design purposes.

\title{
dc and ac behaviour of a normal joint in a superconducting cable
}

\author{
J.L de Reuver, H.H.J. ten Kate, H.G. Knoopers and L.J.M. van de Klundert
}

Koy words: superconductor, ac curront dc current, joint

A superconducting connection between highcurrent cables is hardly possible because of their complicated geometry. Most cables consist of many wires, whilst these wires consist in turn of many filaments. A restriction of a connection between a current supply and its load is the requirement of dismountability. A scale model has been built to investigate the loss behaviour of such joints. Three ways of connecting the cable coupling sockets were tried out. First the connection was performed by applying pressure only, next by introducing a layer of indium between the sockets and finally by soldering the sockets together using Wood's metal

(13.3Sn26.7Pb50Bi10Cd).

After a description of the joint and the used superconducting cable the dc resistances of these connections are shown. Four different methods and their results give rise to some interesting features. The reliability of the methods will be discussed.

The ac behaviour can be described with a model based on the diffusion equation. Results with a ramp and sinusoidal current have also been measured and calculated.

In conclusion the required length of the joint will be derived for ac applications.

\section{Description of the joint}

In order to obtain a high current the joint is included in a closed secondary loop of a transformer (see Fig. 1). Every cable coupling socket consists of a pair of annealed copper blocks soldered to the cable with $\mathrm{Pb} 3 \mathrm{Ag}$. The cable consists of 81 wires in a Litz geometry $(3 \times 3 \times 3 \times 3)$. The cable is filled with $\mathrm{Pb} 40 \mathrm{Sn}$ solder to stiffen it. The strands consist of 114 filaments of NbTi in a Cu30Ni matrix (see Fig. 2). The diameter of the wires and the filaments are 204 and $12.7 \mu \mathrm{m}$ respectively. The matrix to superconductor ratio is 1.25 .

Some remarks in respect to this construction should be made. The high conductivity of the copper sockets $\left(\rho \simeq 3.10^{-10} \Omega \mathrm{m}\right)$ and of $\operatorname{Pb} 3 \mathrm{Ag}\left(\rho \simeq 1.10^{-9} \Omega \mathrm{m}\right)$ reduces the resistance as much as possible. The highly resistive $\mathrm{Cu} 30 \mathrm{Ni}$ matrix material $\left(\rho \simeq 3.810^{-7} \Omega \mathrm{m}\right)$ increases the resistance considerably. However, in a superconducting rectifier flux pump $\mathrm{NbTi} / \mathrm{CuNi}$ wires are used in the thermally activated superconducting switches. A CuNi matrix is preferable in order to obtain a sufficient off-resistance of the switch.

\section{The maximum current}

The maximum obtainable current in the cable in the actual configuration as function of the frequency has been determined using low frequency sinusoidal

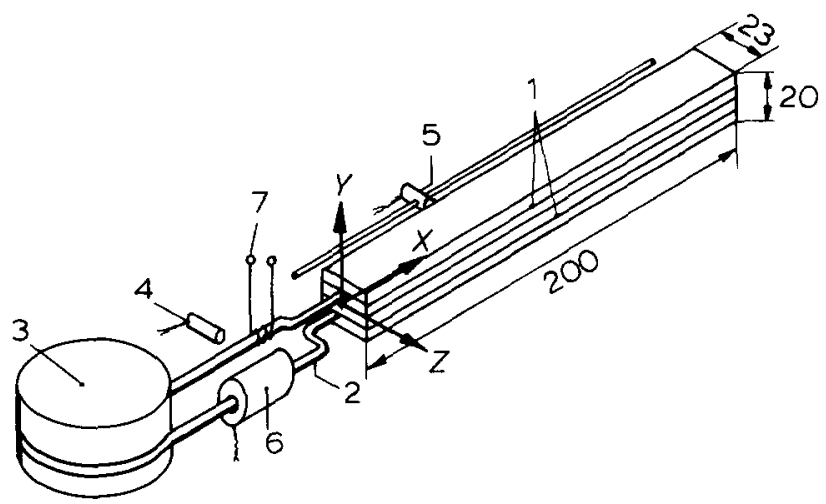

Fig. 1 Configuration of the test set-up. 1 - cable coupling sockets of the joint; 2 - superconducting cable ( $3 \times 3 \times 3 \times 3$ strands); 3 - primary coil: 4 - location of the fixed Hall probe; 5 - support for a Hall probe, movable in the $x$-direction; 6 - Rogowski pick-up coil; 7 - heater 


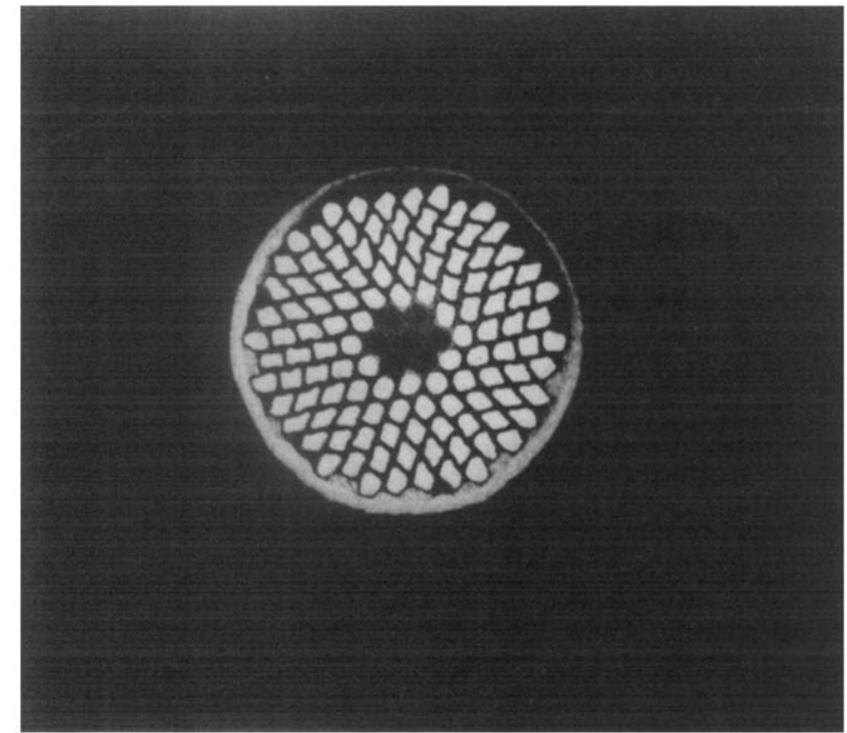

Fig 2 Croes-ection of the NbTVCuNi wire of the MCA 114 filaments diameter $0.204 \mathrm{~mm}$, filament diameter $12.7 \mu \mathrm{m}, \mathrm{CuNi}: \mathrm{c}=1.25: 1$

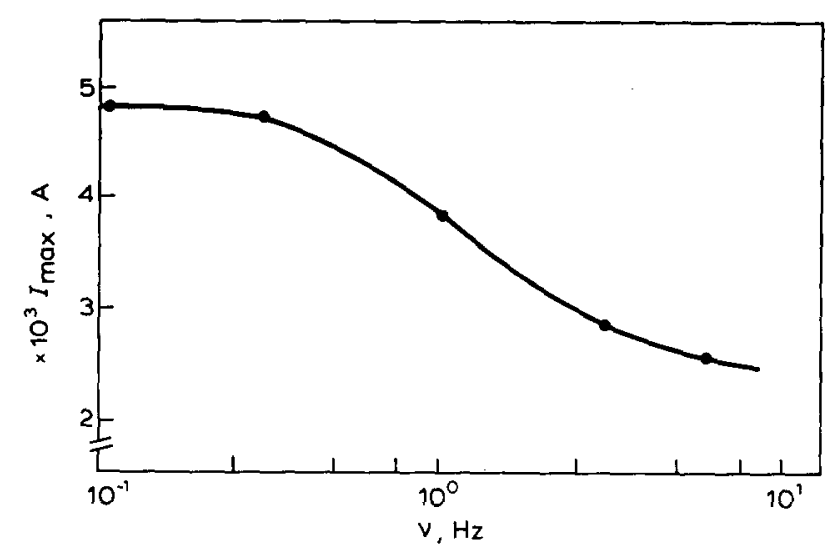

Fig. 3 Maximum obtainable current amplitude versus frequency

currents. We find currents up to about $4800 \mathrm{~A}$ at $0.1 \mathrm{~Hz}$ (see Fig. 3).

In the absence of an external field and when the matrix consists of low conductive material the maximum current will not be equal to the critical current because of instabilities. At higher frequencies a decrease is observed, possibly due to internal heating during ac operation which lowers the critical current density and affects the stability of the wire. These results have been frequently observed in our laboratory in the case of $\mathrm{NbTi} / \mathrm{CuNi}$ wires.

\section{The dc resistance}

Methods to determine the $d c$ resistances. Four methods will be described. Three of them are based on the decay of the current which has been induced in the secondary circuit. Another method is based on the measurement of the voltage across the joint. If the latter becomes more inaccurate in the case of low resistances the current decay technique is preferable.

The current is determined by a fixed Hall probe as shown in Fig. 1. The calibration of the Hall probe is performed by the use of a Rogowski pick-up coil. This can also be used to determine the current directly. A disadvantage of this method is the requirement of large current rates to obtain sufficient accuracy.

To overcome this problem, the detection of the current level at a certain time can be found by changing the state of the superconductor to normal by means of a heater. A typical voltage curve from the pick-up coil as a result of this action is shown in Fig. 4. The integral of this voltage is a measure for the current level.

The method with the Hall probe and the technique with the Rogowski coil yield a decay time coefficient $\tau$. Knowledge of the self-inductance $L$ of the secondary loop is necessary in order to obtain the resistance $R$. Both the self-inductance and the resistance depend on the current:

$$
\tau=\frac{L(I)}{R(I)}
$$

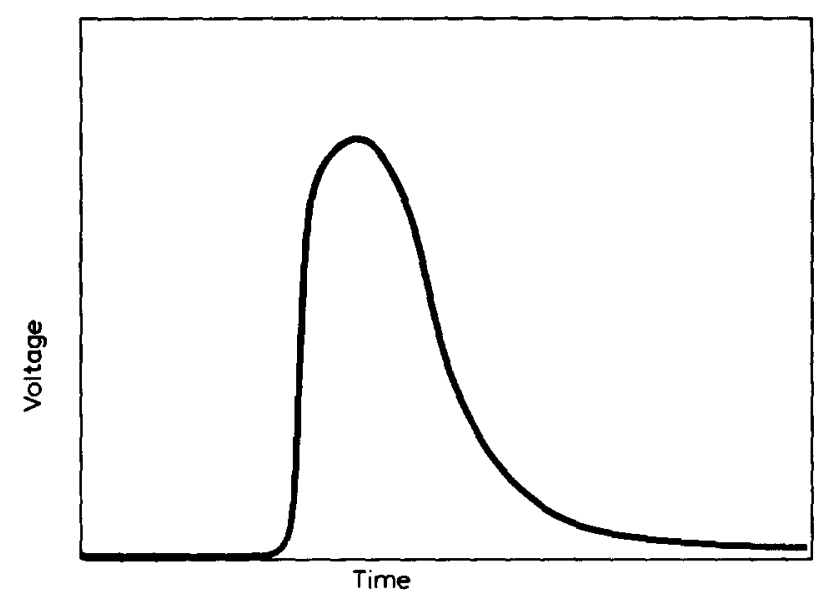

Fig. 4 Rogowski pick-up voltage versus time when the conductor changes into the normal state

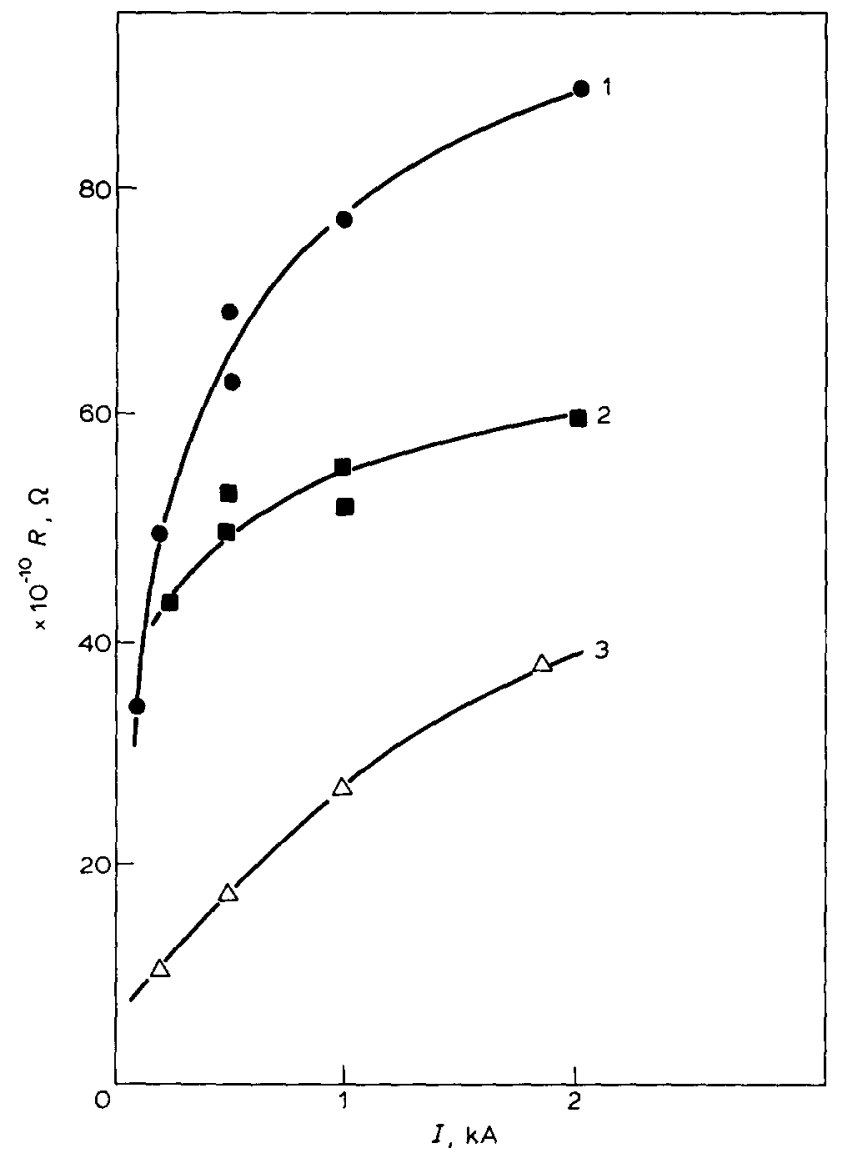

Fig. 5 Joint resistance against current for the various joint configurations. 1 - copper-copper pressure contact; 2 - copper-indium copper pressure contact: 3 - copper-Wood's solder-copper contact 
Another technique to obtain the joint resistance is closely related to the time decay method. During the decay, flux leaves the loop but by means of the primary coil this flux can be pumped into the loop to restore a constant current level (flux pump method). The resistance (3) can be deduced from the transformer equation (2):

$$
\begin{aligned}
& M \dot{I}_{\mathrm{p}}=L I_{\mathrm{s}}+R I_{\mathrm{s}} \\
& R=\frac{M I_{\mathrm{p}}}{I_{\mathrm{s}}}
\end{aligned}
$$

The mutual inductance $(M)$ is now a necessary parameter to find the resistance.

The technical performance is achieved by a feedback system using the Hall voltage whereby the transformer is turned into a current source in this way. This technique is also useful during the direct voltage measurements. A complication that is easy to overcome, is the influence of the stray field of the primary coil. A good orientation of the Hall probe and compensation diminish this influence in a convenient way.

Results of the different joint configurations. In Fig. 5 the results concerning the dc resistance of the coppercopper joint, the copper-indium-copper joint and the copper-Wood's solder-copper joint are shown. This figure is obtained by the Rogowski decay time method. It is clearly shown that a decrease in resistivity takes place in the sequence mentioned of joints.

Adopting a suggestion of Suenaga ${ }^{3}$ an explanation of the decrease using indium is an increase of contact area due to its high ductility compared to copper while Wood's solder guarantees an even larger contact area. As oxide layers are also absent now the solder liquid could affect the resistance in a positive way.

A remarkable feature is the increasing resistance with increasing current. As the cable is transposed completely a current sharing between the wires cannot explain this phenomenon here. Also the current sharing between the filaments in one wire gives no satisfactory explanation. A suggestion, based on the transition of the $\mathrm{Pb} 3 \mathrm{Ag}$ solder from the superconducting to the normal state, is as follows. Taking into account the joint's configuration the magnetic field value decreases along the $x$-axis of the joint. At a certain position about half way in the joint the upper critical field exists. The joint can be roughly divided into two parts with different resistivities. One part has a low conductivity because the solder is normal while the other part has a high conductivity because the solder is superconductive. The value of the current determines the length of each part. It has been possible to deduce a conductivity ratio of 0.37 between the two parts of the joint. A similar influence can be observed if magneto resistance effects play a role.

Comparison of the four measuring techniques. The results, given in Fig. 6, of the direct voltage measurement and the Hall probe decay method agree well, taking into account the accuracy of the determination of the self-inductance being dependent on the current level. The flux pump method systematically gives too high values but for all methods, however, the results are in the same order of magnitude. The Rogowski pick-up coil method gives the least reliable results. In the latter case a discrepancy arises taking into account the initial current level which is included in the figure being caused by the way in which the

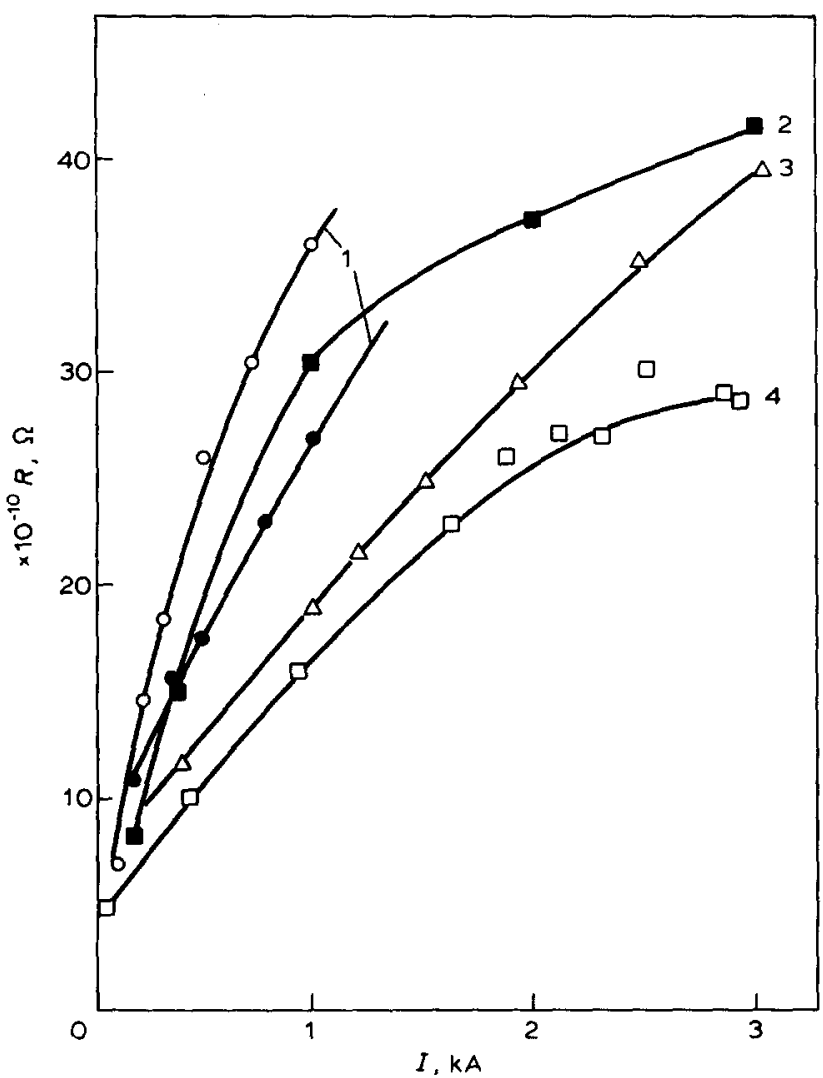

Fig 6 Joint resistance versus current for various measuring methods. 1 - Rogowski pick-up coil decay method, initial current: upper: 1 kA. lower: 4 kA: 2 - flux pump method; 3 - Hall probe decay method

4 - voltage measurement

current is induced. In fact the de current distribution in the joint is not established fast enough. The section on the ac behaviour will clarify this.

\section{The ac resistance}

Experiments have been performed with ramp as well as sinusoidal currents. First a simple onedimensional model will be deduced that covers both cases. The results and the discussion will be treated separately.

Model for the current distribution in the joint. In order to illustrate the phenomena that occur during ac operation, knowledge of the current distribution along the joint is required. It is assumed, for simplicity, that the cables have the same geometry as the joint coupling sockets in the $x-z$ plane (see Fig. 1, for the choice of the axis). The problem is treated onedimensionally and end effects are neglected. The length $(L)$ of the joint has to be introduced for ramp currents while for sinusoidal currents this length may be taken to be infinite as will be seen later. From Ampere's circuital law we can deduce:

$$
B_{\mathrm{z}}(x, t)=\mu_{0} I_{\mathrm{x}}(x, t)
$$

The current occurs as a current per unit of length. Due to the assumptions made earlier the current crosses the joint in the $y$-direction only. Therefore the following holds:

$$
\frac{\partial E_{\mathbf{x}}}{\partial y}=0
$$


(4), (5) combined with Faraday's law give:

$$
\frac{\partial E_{\mathbf{y}}}{\partial x}=-\mu_{0} \frac{\partial I_{\mathbf{x}}}{\partial t}
$$

The conservation of the current is expressed by:

$$
\frac{\partial I_{\mathbf{x}}}{\partial x}=-j_{\mathbf{y}}=-\sigma E_{\mathbf{y}}
$$

A combination of (6) and (7) gives:

$$
\frac{\partial^{2} I_{x}(x, t)}{\partial x^{2}}=\mu_{0} \sigma \frac{\partial I_{x}(x, t)}{\partial t}
$$

This well-known diffusion equation with constant $D=1 / \mu_{0} \sigma$ can be solved using Laplace or Finite Fourier transforms.

Trapezoidal ramp current (see Fig. 7). A direct measurement of this current distribution is very difficult. Voltage terminals along the joint are inconvenient because the equipotential planes are not known sufficiently well. A straight-forward method is to measure the magnetic field at a fixed distance along the joint. The field in the $x$ - and $y$-direction is measured by a movable Hall probe (see Fig. 1). The field pattern can be calculated from the model calculations and a comparison can be made.

The solution of (7) and (8) is given by:

for $t \geq a$

$$
\begin{aligned}
j_{y}(x, t)= & \frac{I_{0}}{L}\left[1+2 \sum_{n=1}^{\infty}\left[\frac{\exp \left(n^{2} \beta a\right)-1}{n^{2} \beta a}\right]\right. \\
& \left.(-1)^{n} \exp \left(-n^{2} \beta t\right) \cos \frac{n \pi(L-x)}{L}\right]
\end{aligned}
$$

for $0 \leq t \leq a$

$$
\begin{aligned}
j_{\mathrm{y}}(x, t)= & \frac{I_{0}}{L}\left[\frac{t}{a}+2 \sum_{n=1}^{\infty}\left[\frac{\exp \left(n^{2} \beta t\right)-1}{n^{2} \beta a}\right]\right. \\
& \left.(-1)^{\mathrm{n}} \exp \left(-n^{2} \beta t\right) \cos \frac{n \pi(L-x)}{L}\right]
\end{aligned}
$$

with

$$
\beta=\frac{\pi^{2} D}{L^{2}}
$$

In Fig. 8 the calculated current distribution is shown. It takes nearly $20 \mathrm{~s}$ to reach a uniform current distribution in the joint and this time should be considered if

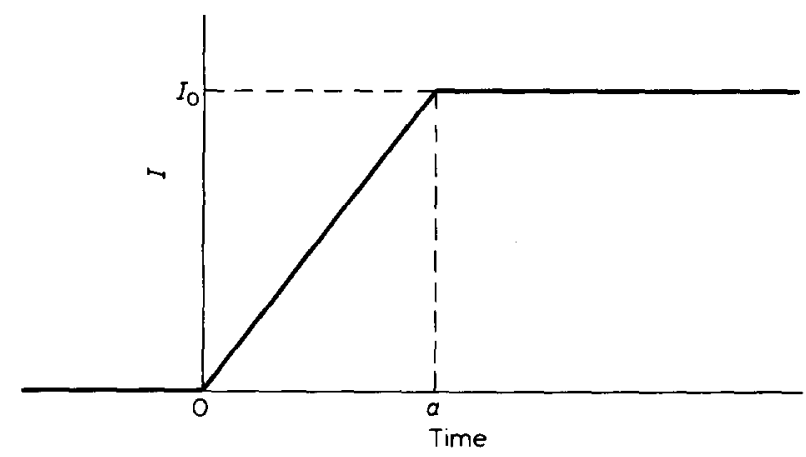

Fig. 7 Shape of ramp current

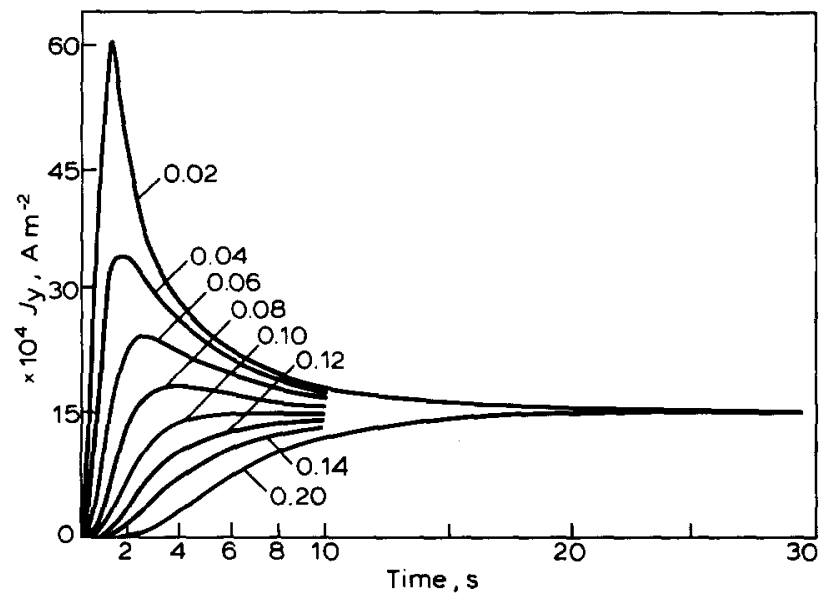

Fig. 8a The calculated current density in the joint when a ramp current is applied with $a=1 \mathrm{~s}$ and $l_{0}=1000 \mathrm{~A}$ for various positions

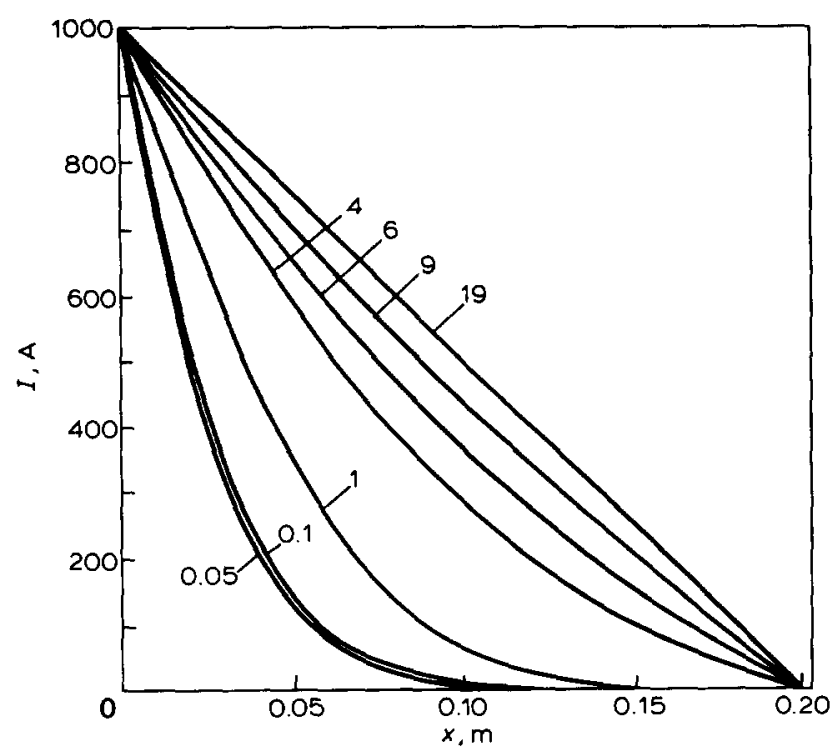

Fig. 8b The calculated current in the cable of the joint versus position for various times according to (7)

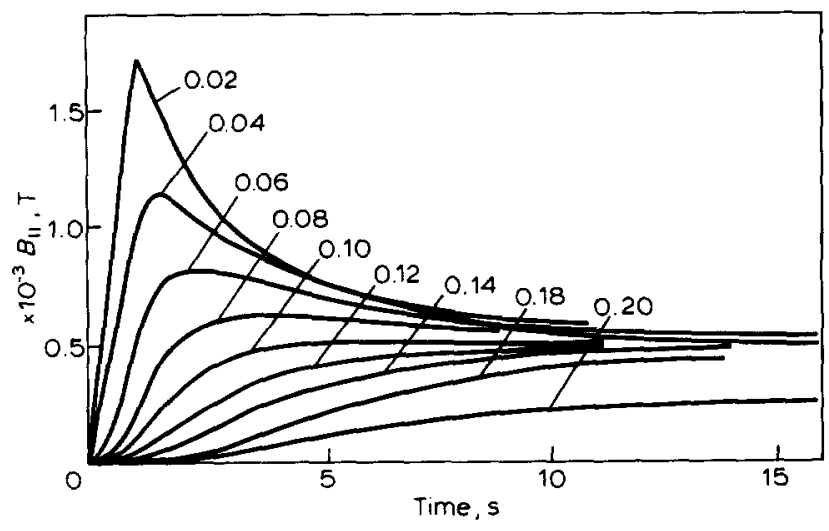

Fig. 9 Calculated paraliel field along the joint for $\theta=1 \mathrm{~s}$ and $I_{0}=2400$ A for various positions

a decay experiment to determine the dc resistance is performed.

Measured and calculated results of the magnetic field parallel to the joint are given in Figs 9 and 10 . The shape of these curves are in good agreement. A difference of a factor of two to obtain quantitative agreement as well, is a consequence of the assumptions in the model. The transition of the solder from the superconductive state to the normal state is of less 


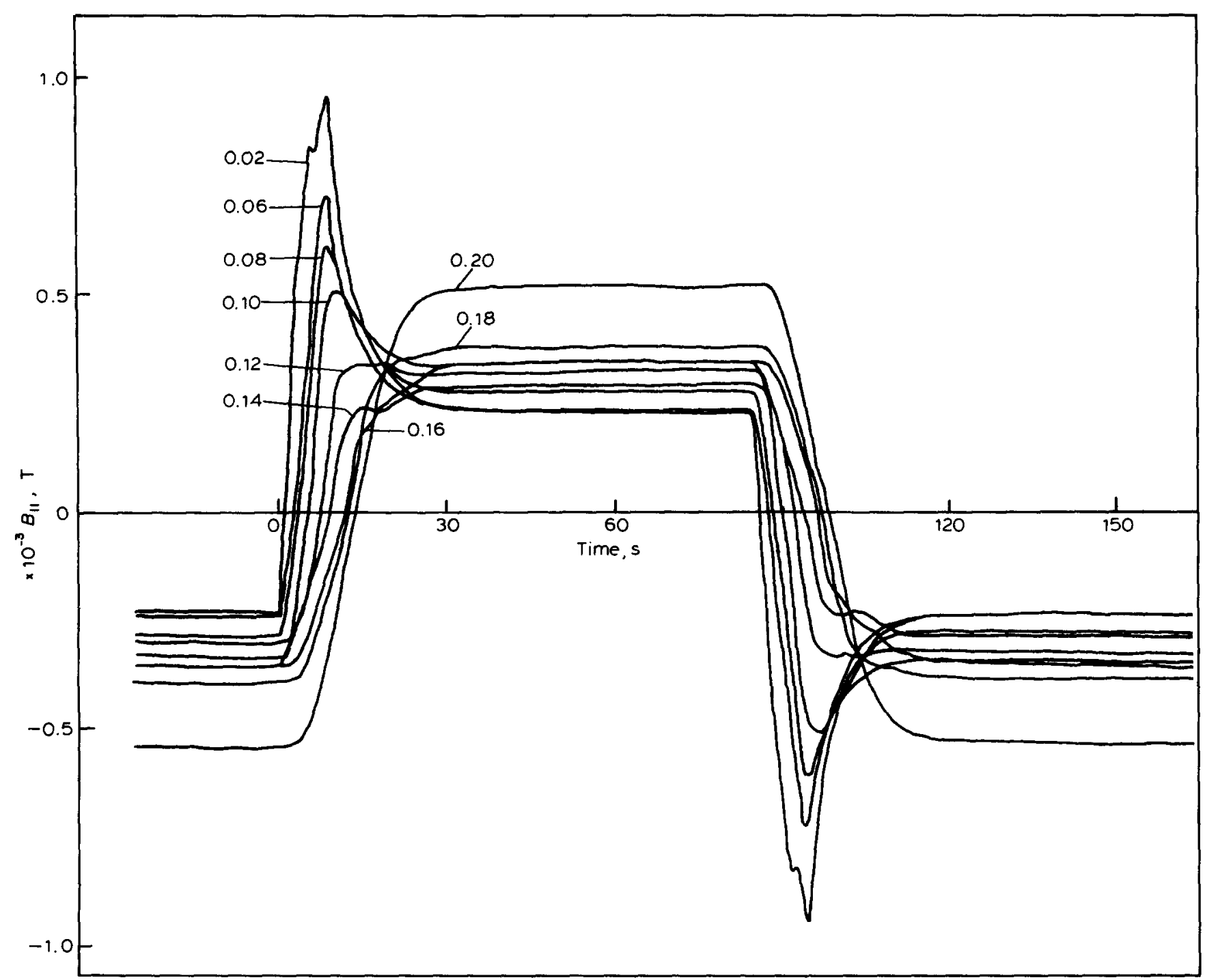

Fig. 10 Measured parallel field along the joint for $a=8 \mathrm{~s}, l_{0}=2400 \mathrm{~A}$ for various positions. The ramp current starts at $I_{0}=-2400 \mathrm{~A}$

interest in ac applications because the part where most of the current crosses from the cable to the other is in the normal state. When a final distribution is reached it is still not homogeneous as can be seen in Fig. 11. The effect of the transition and of the resulting difference in resistivity is the main cause.

Sinusoidal currents. For these currents a periodical solution of $(8)$ is:

$$
I(x, t)=I_{0} \exp (-\alpha x) \cos (\omega t-a x)
$$

with $a=(\omega / 2 D)^{1 / 2}, I_{0}$ the current amplitude, $\omega$ the angular frequency and $D$ the constant of diffusity.

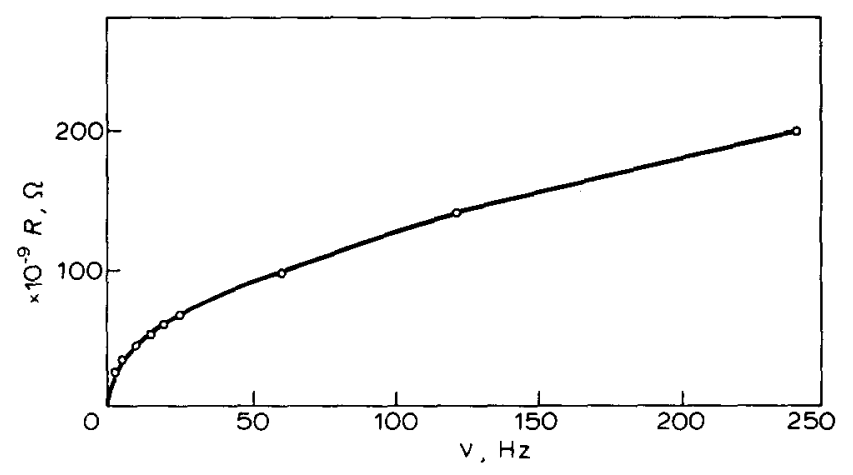

Fig. 11 ac resistance of the joint for sinusoidal currents versus frequency
The current in the loop is:

$$
I(0, t)=I_{0} \cos \omega t
$$

The mean power loss is given by:

$$
\bar{P}=\frac{I_{0}^{2}}{2} \frac{h}{b}\left(\frac{\mu_{0} \omega}{2 \sigma}\right)^{1 / 2}
$$

with $h$ the height and $b$ the width of the joint.

A sort of penetration depth $(p)$ can be deduced from (10) and (12), which gives information on the required length of the joint.

$$
p=\left(\frac{2}{\mu_{0} \omega \sigma}\right)^{1 / 2}
$$

For a frequency of $50 \mathrm{~Hz}$ and a conductivity of $3.10^{9}$ $(\Omega \mathrm{m})^{-1}$ the penetration depth is only $1.3 \mathrm{~mm}$.

In Fig. 11 the ac resistance versus frequency is shown for a current amplitude of $100 \mathrm{~A}$. The voltage has been measured in this case with a lock-in amplifier. 


\section{Conclusions}

The resistance between disassemblable cable coupling sockets can be reduced by increasing the contact area by means of applying indium or Wood's solder. An increase of the resistance with increasing current has been observed. An appropriate explanation for this is the coexistence of superconducting and normal parts in the joint sockets at various fields and currents.

A decay-time technique to obtain the resistance has a good accuracy if notice is taken of the inhomogenity of the current distribution in the joint after the current has been induced. The dependence of the selfinductance and the mutual inductance of the transformer on the current should also be taken into account.

In the case of ac applications, the resistance increases considerably with the square root of the frequency. The magnetic field penetration in the longitudinal direction of the joint determines a useful length of the joint. The construction of an ac joint should be so designed that the occurrence of the diffusion pattern is prevented. The sizes of the joint make it possible to improve the ac resistance, ie the width can be increased instead of the length.
In the case of ramp currents the current distribution is complicated. A measurement technique using a Hall probe that is movable along the joint, can be of great help.

\section{Authors}

The authors are from Twente University of Technology, Department of Applied Physics, PO Box 217, 7500 AE Enschede, The Netherlands. Paper received 5 December 1983.

\section{References}

1 Kate, H.H.J. ten., Bunk, P.B., Steffens, H.A., Klundert, L.J.M. van de A thermally switched $9 \mathrm{kA}$ superconducting rectifier flux pump MAG-17 5 (1981) 2067

2 Kate, H.H.J. ten., Holtslag A.H.M., Steffens, H.A., Knoben, J., Klundert, L.J.M. van de Design of a three phase thermally switched $25 \mathrm{kA}, 1.5 \mathrm{~kW}$ superconducting rectifier flux pump Proc ICEC 9 (1982) 753

3 Suenaga, $\mathbf{M}$. Metallurgical aspects of interstrand resistance Proc US .Japan workshop on superconductive energy storage (1981) 277 